\title{
Angioembolization: Indications, Approach and Optimal Use
}

\author{
Eric N. Klein • Orlando C. Kirton
}

Published online: 10 February 2015

(C) Springer International Publishing AG 2015

\begin{abstract}
Angioembolization has revolutionized the management of pelvic-fracture-related hemorrhage. Along with pelvic immobilization and preperitoneal pelvic packing, angioembolization is an integral component in multidisciplinary algorithms for achieving hemostasis in severe pelvic trauma. Polytrauma, especially associated intraperitoneal injuries, adds to the complexity and complicates decisions related to the optimal care for these patients in hemorrhagic shock. The indications, timing, location, and technique for angioembolization differ based on available resources and expertise. Regional, institutional, and personal preferences add further variation to practice patterns and outcomes. While complications are associated with pelvic angioembolization, the actual causal relationship is questionable. Furthermore, angioembolization is frequently a life-saving maneuver without an effective alternative treatment. The wealth of recent literature will be pieced together to define the role that angioembolization should play in the management of pelvic fracture-related hemorrhage.
\end{abstract}

Keywords Pelvic trauma $\cdot$ Pelvic fracture $\cdot$ Polytrauma Hemorrhagic shock · Angioembolization

\section{Introduction}

In 1977, the "trauma radiologist" was defined as a key member of the trauma team at Kings County Hospital (Brooklyn, NY, USA), actively participating in the diagnosis and

This article is part of the Topical Collection on Trauma to the Pelvis

E. N. Klein • O. C. Kirton $(\square)$

Department of Surgery, Hartford Hospital and the University of

Connecticut School of Medicine, 80 Seymour Street,

Hartford, CT 06102, USA

e-mail: orlando.kirton@hhchealth.org treatment decisions of traumatic hemorrhage. An angiography suite was set up to allow the delivery of critical care during emergency radiologic interventions [1]. Over the past 4 decades, angioembolization has revolutionized how we care for trauma patients. This modality is over $90 \%$ effective in controlling hemorrhage in the abdomen and pelvis from both blunt and penetrating trauma [2]. It is a safe method for hemostasis of surgical bleeding, even in selected hemodynamically unstable patients [3, 4]. Angioembolization is most often used to decrease blood loss and improve success when attempting nonoperative management of hemorrhage, but it can also be used as an adjuvant therapy before, during, or after surgery $[5,6]$. The main applications for angioembolization in trauma patients are of the pelvis, liver, kidneys, and spleen, but this modality has been used for a variety of other injury locations. This article will concentrate on angioembolization of pelvic fracture-related hemorrhage.

Like any surgical treatment, variations exist in the indications and techniques for performing angioembolization in trauma patients. Outcomes differ as a result of these variations, and as evidence by the wealth of publications concentrated over the previous decade, the modality remains in evolution. Much of the pelvic vasculature is intimately associated with bone, and pelvic fractures can lead to vascular injuries (see Fig. 1). Simply opening the retroperitoneum to search for an injured vessel can result in a loss of tamponade and uncontrolled hemorrhage, which can worsen shock, and can lead to the lethal triad of hypothermia, acidosis and coagulopathy, or secondary brain injury. Worst of all, this bloody exploration may still not allow for definitive control of hemorrhage. At the same time, patients with pelvic fractures frequently have associated injuries that can be the cause of additional physiological derangements if unmanaged. Angioembolization has become a key component of damage control laparotomy, an art that has changed the paradigm of trauma care and contributed to a significant drop in mortality [7]. The purpose of this article is to review the literature concerning the indications, 
Fig. 1 Pelvic arterial anatomy. Reproduced with permission from [83]. (Artist: Mr Philippe Payet). http://www.ncbi.nlm.nih.gov/pmc/ articles/PMC2151899. http:// www.biomedcentral.com/about/ license

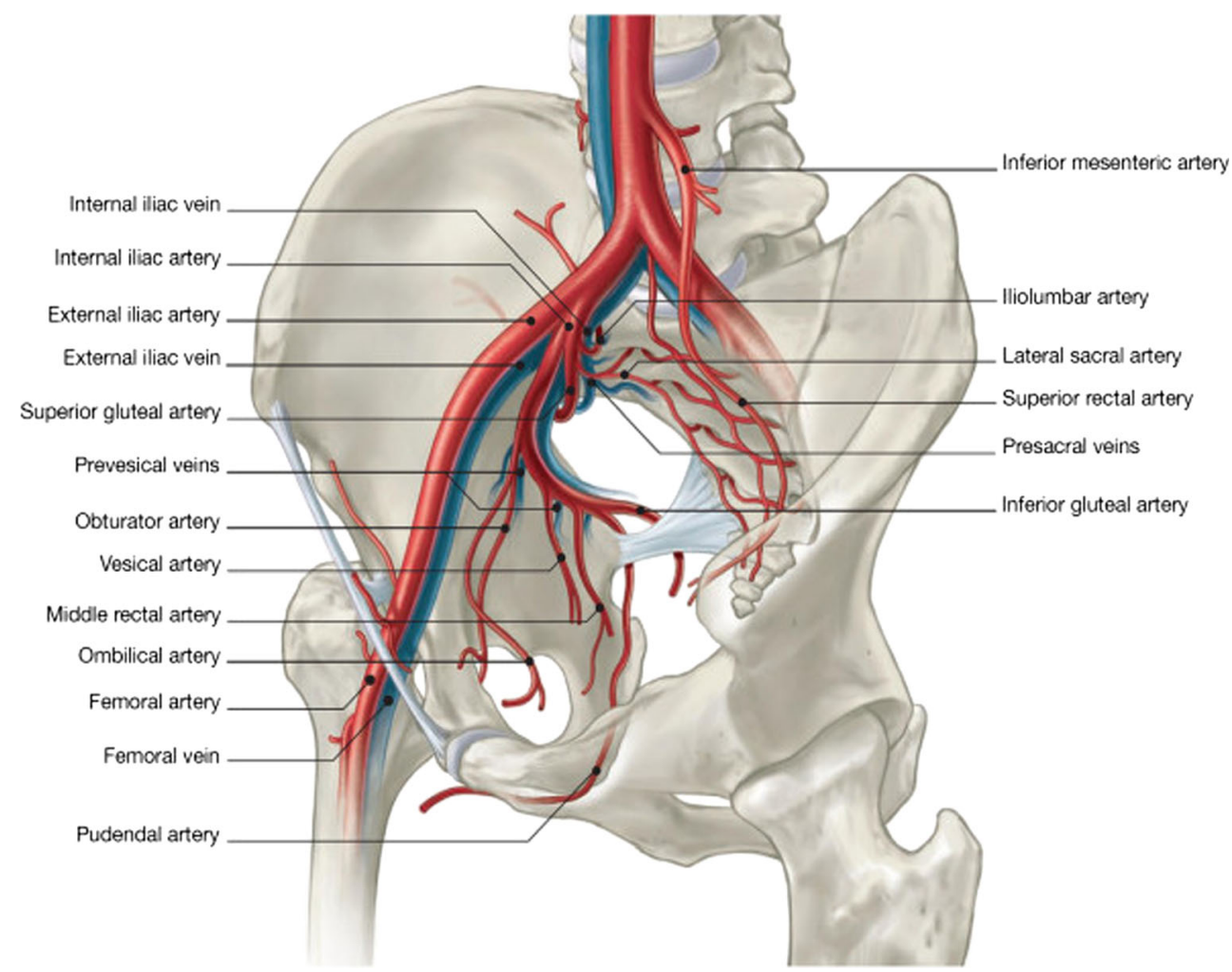

timing, techniques, success rates, and complications of pelvic angioembolization.

\section{Diagnosing Pelvic Fracture-Related Hemorrhage}

Advanced Trauma Life Support (ATLS) management algorithm provides quick diagnostic tools for all sources of traumatic hemorrhage. During resuscitation of the hypotensive patient, these should be carried out expeditiously in anticipation of having to control hemorrhage in a nonresponder or transient responder [8]. Sometimes, we are lucky to find only a single potential source of hemorrhage, and implementation of an effective method for hemostasis can save the patient's life. In other patients, we find multiple potential sources and face the dilemma of determining the most significant cavity that must be tackled first.

Unstable pelvic fractures can be diagnosed on physical exam or plain X-ray. There is much debate on the need for routine pelvic X-rays in all blunt trauma patients, especially in the era when most patients undergo a pelvic CT scan. Pelvic $\mathrm{CT}$ is more sensitive for fractures than X-rays, but more time consuming [9-11]. In an early study, an unstable fracture on pelvic X-ray was $100 \%$ sensitive for detecting pelvic arterial injuries. In that study, unstable fractures were defined as either an anterior-posterior (AP) compression fracture or $>0.5 \mathrm{~cm}$ of bony displacement [10]. In a more recent study, patients with unstable pelvic fractures (AP type II/III, LC type II/III, VS) on
X-ray were found to require a greater volume of blood transfusion and were more likely to require angioembolization [11]. Pelvic X-ray can even predict the location and laterality of hemorrhage site with nearly perfect sensitivity for anterior sites and perfect specificity for posterior sites. This information can help with the identification of the initial candidate vessels for selective angioembolization [12].

Some patients with pelvic fractures are stable enough for CT scan. Contrast blush on a 64-slice CT scan is $82 \%$ sensitive and $95 \%$ specific in diagnosing pelvic fracture-related hemorrhage that requires intervention [13]. Contrast blush on CT scan is highly predictive of arterial extravasation on angiography $[14,15]$, especially in the setting of hemodynamic instability [16-18]. Nonetheless, some hemodynamically unstable patients will present with a pelvic fracture without associated hematoma or contrast blush on CT scan, yet still demonstrate extravasation on angiography; and can frequently benefit from angioembolization [19, 20]. Furthermore, even patients with stable pelvic fracture patterns (AP type I, LC type I/II) may have arterial injuries on angiography, especially those with signs of ongoing hemorrhage [21]. Sacroiliac joint disruption on either X-ray or CT scan and duration of hypotension were each found to be independent predictors of contrast extravasation on angiogram [22]. The location of contrast blush and large pelvic hematomas on CT can be used to predict the injured artery and guide selective embolization (see Table 1) [23-25]. Anterior-posterior compression fractures place patients at increased risk for bilateral posterior arterial 
Table 1 Arterial injuries related to fractures

\begin{tabular}{ll}
\hline Fracture & Artery \\
\hline Sacroiliac joint & Iliolumbar artery \\
Sacrum & Lateral sacral artery \\
Iliac bone & Superior gluteal artery \\
& Inferior gluteal artery \\
Acetabulum & Obturator artery \\
& Superior gluteal artery \\
& Inferior gluteal artery \\
Pubic rami & Internal pudendal artery \\
& Obturator artery \\
\hline
\end{tabular}

injuries, even when only unilateral blush is seen on CT scan. These patients should undergo bilateral internal iliac artery angiograms [26].

Pelvic fractures are common among victims of blunt trauma, but very few of these patients require angioembolization. In hemodynamically unstable trauma patients, the pelvis must be evaluated for potential hemorrhage. If physical exam does not demonstrate an unstable pelvis, imaging must be obtained. The pelvic X-ray is an expedient technique to evaluate for pelvic fracture-related hemorrhage that may require intervention. When a CT scan is available, contrast blush can further predict patients who might benefit angioembolization. While the studies mentioned above demonstrate which patients might have benefited from angioembolization, they are not able to evaluate whether alternative methods for control of pelvic fracture-related hemorrhage could be as or more effective.

\section{Controlling Pelvic Fracture-Related Hemorrhage}

There are multiple options for controlling pelvic-fracturerelated hemorrhage. Pelvic immobilization can be performed with a pelvic binder, a C-clamp, or external fixation. These techniques decrease the pelvic volume, permits tamponade to occur, and allows clot to stabilize, which decreases the bleeding from fractured bony surfaces. Please refer to the previous article "Orthopedic Approach to the Early Management of Pelvic Injuries" for more details about these procedures. Preperitoneal pelvic packing has been popular in Europe for years and is now gaining support in American trauma centers. Please refer to the following article "Pre-peritoneal Packing: How and When" for more details about this procedure. Along with angioembolization, these techniques have overlapping and complementary, but sometimes competing roles in controlling pelvic fracture-related hemorrhage. It is important to develop a standardized multidisciplinary approach to pelvicfracture-related hemorrhage. Implementing an institutional practice guideline to coordinate care has led to a decrease in transfusion requirements and mortality [27]. Refer to Fig. 2 for the algorithm included in our guidelines for the management of patients with pelvic fractures and hemodynamic compromise.

In one study of adults, pelvic stabilization controlled hemorrhage in $85 \%$ of patients, and angioembolization controlled hemorrhage in all the remaining patients [28]. Older patients (over the age of 55 or 60) have been shown to experience greater benefit from angioembolization than younger patients [29, 30]. Pelvic fractures are less common among pediatric trauma patients than adults. In the pediatric population, fracture pattern is strongly correlated to the risk of hemorrhage, with bilateral anterior and posterior fractures having the highest risk. In a study of pediatric patients, skeletal fixation was effective at controlling hemorrhage in $60 \%$ of patients, and angioembolization controlled hemorrhage in $75 \%$ of remaining children [31]. The survival rate after successful angioembolization has been estimated to over $80 \%$, and rate of transfusion prior to the procedure predicted mortality in a multivariate regression model [32].

Pelvic stabilization should be the first maneuver for controlling pelvic fracture-related hemorrhage [33]. The pelvic binder is a quick and simple method to stabilize the pelvis. It may be applied to all patients who are suspected to be suffering from pelvic fracture-related hemorrhage, and while it may not benefit all types of fractures, it is unlikely to cause significant harm if applied properly around the greater trochanters. A C-clamp can also be applied in the trauma resuscitation area, though not as quickly. Approximately one third of patients will remain hemodynamically unstable after pelvic stabilization with a C-clamp and necessitate angioembolization [34]. While the C-clamp has the added theoretical benefit of stabilizing the posterior pelvis wherein lies the rich venous plexus and large bony surfaces, the pelvic binder seems to have similar effectiveness, and this is our preferred method at Hartford Hospital [35]. Operative external fixation is also an effective method to stabilize the pelvis, but takes much longer than application of a pelvic binder or C-clamp and seems to have similar benefits. Therefore, in hemodynamically unstable patients, operative external fixation should be delayed until after angioembolization is complete [36, 37]. In hemodynamically stable patients with evidence of ongoing hemorrhage and an unstable pelvic fracture, operative external fixation may be preferred over angioembolization.

Preperitoneal pelvic packing combined with external fixation has been shown to decrease pelvic fracture-related hemorrhage in hypotensive patients with pelvic fractures. External fixation was performed with either a C-clamp or external fixator. Only $13 \%$ of patients went on to require angioembolization, and it was performed over $10 \mathrm{~h}$ after admission on average [38••]. A similar protocol in which angioembolization is performed when preperitoneal pelvic 


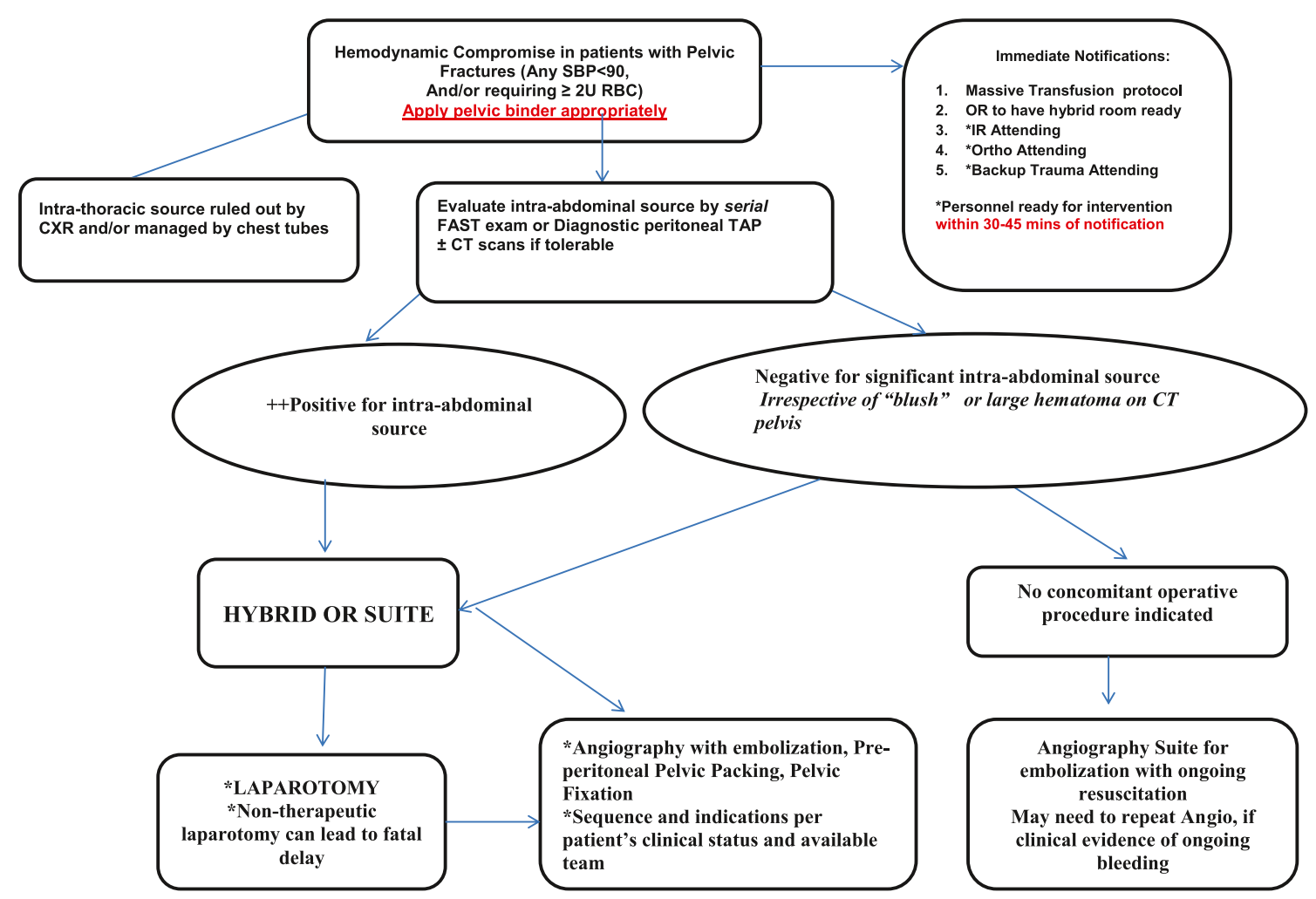

Fig. 2 Hartford hospital algorithm for the multidisciplinary management of patients with pelvic fractures and hemodynamic compromise

packing and external fixation fail to control hemorrhage is similarly effective in both the adult [39] and pediatric trauma population [40]. When patients have persistent hemorrhage after pelvic stabilization, preperitoneal pelvic packing seems to be more effective at controlling pelvic fracture-related hemorrhage than angioembolization, but this could be a result of the fact that angioembolization was performed an average of 85 min later than preperitoneal pelvic packing [41]. Delay in pelvic embolization has been shown to negatively affect patients because of ongoing blood loss [42, 43]. Admission during the night is associated with a 90 -min delay in angioembolization for pelvic fractures, and this delay is estimated to nearly double the mortality [44•]. A trauma center in Tokyo has made fluoroscopy available in the trauma resuscitation room and trained their surgeons to perform diagnostic and therapeutic angiography. This intervention has decreased the mortality in patients with pelvic fracture hemorrhage and other sources of bleeding, likely due to earlier hemostasis and less blood loss [45••, 46].

In the hemodynamically unstable patient with pelvicfracture-related hemorrhage as the sole cause, a pelvic binder should be placed. If the patient remains hemodynamically unstable, preperitoneal pelvic packing should be performed, followed by angioembolization as necessary. There are a variety of indications for pelvic angioembolization, although none are absolute (see Table 2). For persistent hemodynamic lability, it is essential to repeat a focused assessment with sonography for trauma (FAST) exam or perform a supraumbilical diagnostic peritoneal aspiration.

\section{Concomitant Intraperitoneal Hemorrhage}

Pelvic fractures, especially lateral compression patterns, are often associated with concomitant intra-abdominal injuries because they often result from high energy mechanisms. There are multiple options for diagnosing intraperitoneal injuries (see Table 3). Coexistence of hemoperitoneum and a pelvic fracture in a hemodynamically unstable trauma patient creates a difficult decision for the surgeon. Emergent laparotomy can provide rapid definitive control of intraperitoneal hemorrhage; however, it can still be a nontherapeutic maneuver. Laparotomy rarely allows for successful control of pelvicfracture-related hemorrhage. In a multicenter study of hemodynamically unstable patients with pelvic fractures in which

Table 2 Indications for angiography for pelvic fractures

Hemodynamic instability

Contrast blush on CT scan

Large hematoma on CT scan

Persistent hemorrhage in a stable (or stabilized) pelvis

Recurrent hemorrhage after initial angioembolization

Expanding gluteal hematoma 
Table 3 Methods to diagnose intraperitoneal injury

\begin{tabular}{ll}
\hline FAST & Quick \\
& Less sensitive \\
$\begin{array}{l}\text { Diagnostic peritoneal } \\
\text { aspiration }\end{array}$ & Quick (must be done \\
CT scan & above the umbilicus) \\
& Time consuming \\
& Most informative \\
\hline
\end{tabular}

109 underwent laparotomy, $24 \%$ were found to have no intraabdominal injury, and an additional $49 \%$ were found to have no active intraperitoneal hemorrhage. As such, laparotomy was nontherapeutic in $73 \%$ of these patients. Of note, $20 \%$ of these patients who had undergone nontherapeutic laparotomy had no preoperative study performed to show intraperitoneal hemorrhage (FAST, DPL, and CT scan), so this timeconsuming surgery could have potentially been avoided in some of them [47]. In another study, the rate of nontherapeutic laparotomies in patients with severe pelvic fractures was found to be $38 \%$. Two of these patients underwent laparotomy despite having a CT scan with no evidence of intraperitoneal hemorrhage, again, suggesting that, with proper workup, the rate of nontherapeutic laparotomy would be lower [48], and in a third study of patients with pelvic-fracture-related hemorrhage, FAST exam within 15 min of ED arrival had a $97 \%$ positive predictive value for significant abdominal injury leading to therapeutic laparotomy [49]. In patients who have a CT scan, the size of hemoperitoneum, based on how many areas of the abdomen contain free fluid, can further discriminate the need for laparotomy $[50 \bullet, 51]$.

Angioembolization is effective in controlling pelvicfracture-related hemorrhage and frequently intraperitoneal hemorrhage, but it may not be the quickest method for controlling intraperitoneal hemorrhage [52]. Even in hemodynamically unstable patients with multicompartment hemorrhage, angioembolization might benefit patients more than surgery by avoiding further tissue damage and the resulting physiologic insult while effectively controlling hemorrhage $[53 \cdot, 54]$. While many patients with unstable pelvic fracture patterns may benefit from undergoing angiography prior to laparotomy for concomitant hemoperitoneum [6, 55], select patients seem to require laparotomy prior to pelvic angiography. Fang found that patients with a pelvic hematoma and any of the following four findings on CT scan should have pelvic angiography delayed until after laparotomy: major mesenteric tear with contrast blush, a large contrast blush from intraperitoneal solid organs in patients who do not respond to fluid resuscitation, a massive diaphragm rupture with respiratory compromise, and contrast blush from vessels inaccessible to angioembolization [56]. Others recommend performing laparotomy prior to angiography in all patients with hemoperitoneum and persistent hemorrhage despite pelvic stabilization [57]. Taking into account the delay until angioembolization can be performed, these patients may benefit from intraoperative bilateral internal iliac artery ligation as a damage-control technique to control exsanguinating pelvicfracture-related hemorrhage [58]. Preperitoneal pelvic packing through a separate incision is an alternative technique that can be performed at the same time as laparotomy [59].

When having to choose between the operating room for laparotomy or the angiography suite for pelvic embolization, an incorrect decision can have fatal consequences for the patient [60]. Angioembolization can be safely performed in the operating room where other interventions are also possible and avoids the potential delay from transport to or from the radiology suite [61]. Specialized hybrid operating rooms are being designed to accommodate the multidisciplinary treatment strategies for trauma patients [62・•], but angioembolization using a portable $\mathrm{C}$-arm is safe and effective [63]. In hemodynamically unstable patients with evidence of both intraperitoneal and pelvic fracture-related hemorrhage, our practice is to place a pelvic binder and proceed emergently to our hybrid operating room (see Fig. 2). This guarantees that we will have optimal radiological equipment and that angioembolization supplies are immediately available. We begin by performing a damage control laparotomy. If the patient does not stabilize immediately upon controlling any sources of intraperitoneal hemorrhage, we proceed to preperitoneal pelvic packing. We employ angioembolization liberally and prefer to have an external fixation device placed if the fracture seems unstable, but these last two steps vary based on hemodynamic stability and concern for significant head injury.

\section{Angioembolization Techniques}

Angioembolization has revolutionized the treatment of pelvicfracture-related hemorrhage. Hemorrhage from the maze of inaccessible branching vessels along the pelvic sidewall and sacral hollow was associated with significant mortality prior to advances in angioembolization. The earliest reports from the 1970s described selective embolization using clotted autologous blood as the coagulation agent [64-66]. Practice shifted to using gelfoam for arterioles and capillary beds [67] and metallic coils, sometimes in combination with gelfoam, to occlude arteries [68, 69]. The signs of arterial injury on angiography include contrast extravasation, pseudoaneurysm, arteriovenous fistula, abrupt cutoff of flow, and vessel narrowing not associated with a bifurcaton. The use of subselective embolization should be limited to hemodynamically stable patients.

In 2000, Velmahos described the damage-control technique of nonselective bilateral internal iliac artery embolization using gelfoam. This was used to provide temporary occlusion when the hemorrhage source was diffuse from multiple 
branches bilaterally, when hemodynamic lability prevented selecting multiple branches for embolization, or when the patient required continued transfusion after apparently successful subselective embolization. In this series, overall success was $90 \%$ and included repeat angioembolization in two thirds of patients in whom it initially failed [70]. Nonselective bilateral internal iliac artery embolization should be the procedure of choice in hemodynamically unstable patients.

\section{Angioembolization Complications}

Catheter-related complications can occur at the access point or from catheter manipulation [3, 71]. These vascular complications are not unique to angiography in trauma patients. They are uncommon, and their treatment varies broadly from observation to revascularization. Inadvertent embolization of the femoral artery and subsequent acute limb ischemia was noticed early on as a complication of pelvic embolization [67]. Pelvic trauma is a known risk for sexual dysfunction, and angioembolization has not been shown to increase this complication [72]. It has, however, been associated with increased rates of buttock, thigh, or perineal paresthesia [73].

Gluteal necrosis has been reported after nonselective internal iliac artery embolization, but this complication does not seem to occur after selective embolization [74]. A retrospective study described six patients with gluteal necrosis, four had bilateral nonselective internal artery embolization, and two had bilateral superior and inferior gluteal artery embolization. Half the patients died as a result. Compression from orthopedic stabilization devices distal to the embolized vessel was noted as a contributing cause to ongoing hemorrhage in two of the patients [75]. Another retrospective study found that all patients with complications after angioembolization had had bilateral embolization, and all patients with gluteal necrosis had undergone nonselective embolization. The study describes performing nonselective embolization at or above the level of the internal iliac arteries. It does not explain the reason for performing embolization proximal to the internal iliac arteries [76]. Neither of these articles discussed whether coils were used for embolization or only gelfoam, and it is difficult to discern whether they resulted from angioembolization or if the initial trauma actually caused the complications.

Contrast nephropathy is a risk of angiography; concomitant hypotension and renal hypoperfusion can exacerbate this risk. Sauer compared rates of acute kidney injury (AKI) in patients with high-grade renal trauma based on whether or not they underwent angioembolization. Despite higher grade injuries in the group undergoing angioembolization, there was no difference in rates of AKI between the groups. Only $10 \%$ of patients still had mild AKI at the time of discharge, and none required renal replacement therapy [77]. Contrast nephropathy is more common in patients with renal injury than with other organ injuries, but it is still transient and resolves in just a few days [78].

Coil migration has been reported as a complication that can contribute to rebleeding after successful embolization [79]. Gelfoam provides only temporary occlusion and dissolves within days (sometimes hours), potentially resulting in recurrent bleeding. Repeat angioembolization is successful in treating patients with evidence of recurrent bleeding after primary angioembolization for pelvic fracture-related hemorrhage [80-82]. Despite multiple reported complications after angioembolization, many could be attributed to the high burden of traumatic injury. When employed to control potentially life-threatening hemorrhage, the frequent benefit surely outweighs the rare risk.

\section{Conclusions}

No randomized controlled trials exist addressing the use of angioembolization in trauma patients. The modality was initially attempted to control intraperitoneal solid organ, retroperitoneal and pelvic fracture-related hemorrhage when surgical options were dismal. Success was quickly apparent, techniques evolved, and innovations were reported in case series and and single-institution experiences. As techniques advanced and clinical experience progressed, surgeons and radiologists have attempted to define algorithms and protocols for the optimal applications of angioembolization in trauma patients. Their success has been thwarted by the infinite combinations of traumatic injuries and delayed presentations faced by their subjects. We are left with a myriad of articles often with a conclusion that contradicts published recommendations based upon another center's experience. Despite the lack of guideline consensus and frequent protocol deviations in multiple published studies, no trauma surgeon can dispute the fact that angioembolization has revolutionized the care of trauma patients, especially those with pelvic fracture-related hemorrhage. It is a tribute of successful multidisciplinary cooperation that has fostered improvements in patient survival over the past 4 decades.

\section{Compliance with Ethics Guidelines}

Conflict of Interest Eric N. Klein and Orlando C. Kirton declare that they have no conflict of interest.

Human and Animal Rights and Informed Consent This article does not contain any studies with human or animal subjects performed by any of the authors. 


\section{References}

Papers of particular interest, published recently, have been highlighted as:

- Of importance

- Of major importance

1. Sclafani SJ et al. Interventional radiology in trauma victims: analysis of 51 consecutive patients. J Trauma. 1982;22(5):353-60.

2. Velmahos GC, et al. Angiographic embolization for intraperitoneal and retroperitoneal injuries. World J Surg. 2000;24(5):539-45.

3. Velmahos GC, et al. A prospective study on the safety and efficacy of angiographic embolization for pelvic and visceral injuries. $\mathrm{J}$ Trauma. 2002;53(2):303-8. discussion 308.

4. Hagiwara A, et al. The usefulness of transcatheter arterial embolization for patients with blunt polytrauma showing transient response to fluid resuscitation. J Trauma : Inj Infect Crit Care. 2004;57(2):271-7.

5. Velmahos GC, et al. Angiographic embolization for arrest of bleeding after penetrating trauma to the abdomen. Am J Surg. 1999;178(5):367-73.

6. Kushimoto $\mathrm{S}$, et al. The role of interventional radiology in patients requiring damage control laparotomy. J Trauma. 2003;54(1):171-6.

7. Hoffer EK, et al. Endovascular techniques in the damage control setting. Radiographics. 1999;19(5):1340-8.

8. Subcommittee, A., T. American College of Surgeons' Committee on, and A.w.g. International. Advanced trauma life support (ATLS(R)): the ninth edition. J Trauma Acute Care Surg. 2013;74(5):1363-6.

9. Guillamondegui OD, et al. Pelvic radiography in blunt trauma resuscitation: a diminishing role. J Trauma. 2002;53(6):1043-7.

10. Cryer HM, et al. Pelvic fracture classification: correlation with hemorrhage. J Trauma. 1988;28(7):973-80.

11. $\mathrm{Fu} \mathrm{CY}$, et al. Evaluation of pelvic fracture stability and the need for angioembolization: pelvic instabilities on plain film have an increased probability of requiring angioembolization. Am J Emerg Med. 2009;27(7):792-6

12. Niwa $T$, et al. The value of plain radiographs in the prediction of outcome in pelvic fractures treated with embolisation therapy. Br J Radiol. 2000;73(873):945-50.

13. Mohseni S, et al. The diagnostic accuracy of 64-slice computed tomography in detecting clinically significant arterial bleeding after pelvic fractures. Am Surg. 2011;77(9):1176-82.

14. Stephen DJ, et al. Early detection of arterial bleeding in acute pelvic trauma. J Trauma. 1999;47(4):638-42.

15. Brasel KJ, et al. Significance of contrast extravasation in patients with pelvic fracture. J Trauma. 2007;62(5):1149-52.

16. Pereira SJ, et al. Dynamic helical computed tomography scan accurately detects hemorrhage in patients with pelvic fracture. Surgery. 2000;128(4):678-85.

17. Brun J, et al. Detecting active pelvic arterial haemorrhage on admission following serious pelvic fracture in multiple trauma patients. Injury. 2014;45(1):101-6.

18. Verbeek DO, et al. Management of pelvic ring fracture patients with a pelvic "blush" on early computed tomography. J Trauma Acute Care Surg. 2014;76(2):374-9.

19. Brown CV, et al. Does pelvic hematoma on admission computed tomography predict active bleeding at angiography for pelvic fracture? Am Surg. 2005;71(9):759-62.

20. $\mathrm{Fu} \mathrm{CY}$, et al. Angioembolization provides benefits in patients with concomitant unstable pelvic fracture and unstable hemodynamics. Am J Emerg Med. 2012;30(1):207-13.
21. Sarin EL, et al. Pelvic fracture pattern does not always predict the need for urgent embolization. J Trauma : Inj Infect Crit Care. 2005;58(5):973-7.

22. Salim A et al. Predictors of positive angiography in pelvic fractures: a prospective study. J Am Coll Surg. 2008;207(5): $656-62$.

23. Hallinan JT, Tan $\mathrm{CH}$, Pua U. Emergency computed tomography for acute pelvic trauma: where is the bleeder? Clin Radiol. 2014;69(5): 529-37.

24. Cerva Jr DS, et al. Detection of bleeding in patients with major pelvic fractures: value of contrast-enhanced CT. AJR Am J Roentgenol. 1996;166(1):131-5.

25. Dormagen JB, et al. Efficacy of plain radiography and computer tomography in localizing the site of pelvic arterial bleeding in trauma patients. Acta Radiol. 2010;51(1):107-16.

26. $\mathrm{Fu} \mathrm{CY}$, et al. Anterior-posterior compression pelvic fracture increases the probability of requirement of bilateral embolization. Am J Emerg Med. 2013;31(1):42-9.

27. Balogh $Z$, et al. Institutional practice guidelines on management of pelvic fracture-related hemodynamic instability: do they make a difference? J Trauma. 2005;58(4):778-82.

28. Metsemakers WJ, et al. Transcatheter embolotherapy after external surgical stabilization is a valuable treatment algorithm for patients with persistent haemorrhage from unstable pelvic fractures: outcomes of a single centre experience. Injury. 2013;44(7):964-8.

29. Kimbrell BJ, et al. Angiographic embolization for pelvic fractures in older patients. Arch Surg. 2004;139(7):728-32. discussion 732-3.

30. Henry SM, et al. Pelvic fracture in geriatric patients: a distinct clinical entity. J Trauma. 2002;53(1):15-20.

31. McIntyre Jr RC, et al. Pelvic fracture geometry predicts risk of life-threatening hemorrhage in children. J Trauma. 1993;35(3):423-9.

32. Wong YC, et al. Mortality after successful transcatheter arterial embolization in patients with unstable pelvic fractures: rate of blood transfusion as a predictive factor. J Trauma. 2000;49(1):71-5.

33. Abrassart S, Stern R, Peter R. Unstable pelvic ring injury with hemodynamic instability: what seems the best procedure choice and sequence in the initial management? Orthop Traumatol Surg Res. 2013;99(2):175-82.

34. Sadri H, et al. Control of severe hemorrhage using C-clamp and arterial embolization in hemodynamically unstable patients with pelvic ring disruption. Arch Orthop Trauma Surg. 2005;125(7): 443-7.

35. Pizanis A, et al. Emergency stabilization of the pelvic ring: clinical comparison between three different techniques. Injury. 2013;44(12):1760-4.

36. Miller PR, et al. External fixation or arteriogram in bleeding pelvic fracture: initial therapy guided by markers of arterial hemorrhage. J Trauma. 2003;54(3):437-43.

37. Tanizaki S, et al. Early embolization without external fixation in pelvic trauma. Am J Emerg Med. 2012;30(2):342-6.

$38 . \bullet$ Burlew CC, et al. Preperitoneal pelvic packing/external fixation with secondary angioembolization: optimal care for lifethreatening hemorrhage from unstable pelvic fractures. J Am Coll Surg. 2011;212(4):628-35; discussion 635-7. This article describes a multidisciplinary approach that is effective at stopping hemorrhage in severely injured patients (injury severity score= 52) with pelvic fracture related hemorrhage. Despite suffering from hemorrhagic shock, the overall mortality was only $21 \%$ and no patient died of exsanguination.

39. Cheng $\mathrm{M}$, et al. Improvement in institutional protocols leads to decreased mortality in patients with haemodynamically unstable pelvic fractures. Emerg Med J. 2013. doi: 10.1136/emermed2012-202009 
40. Chao NS, et al. Retroperitoneal pelvic packing for haemodynamically unstable pelvic fractures in children and adolescents: a levelone trauma-centre experience. J Pediatr Surg. 2012;47(12): 2244-50.

41. Osborn PM, et al. Direct retroperitoneal pelvic packing versus pelvic angiography: a comparison of two management protocols for haemodynamically unstable pelvic fractures. Injury. 2009;40(1): 54-60.

42. Hauschild O, et al. Angioembolization for pelvic hemorrhage control: results from the German pelvic injury register. J Trauma Acute Care Surg. 2012;73(3):679-84.

43. Tanizaki $\mathrm{S}$ et al. Time to pelvic embolization for hemodynamically unstable pelvic fractures may affect the survival for delays up to 60 min. Injury. 2014;45(4):738-41.

44. - Schwartz DA, et al. Are we delivering two standards of care for pelvic trauma? Availability of angioembolization after hours and on weekends increases time to therapeutic intervention. J Trauma Acute Care Surg. 2014;76(1):134-9. This paper demonstrates an increased mortality because of delay in the availability of angioembolization during nights and weekends. Achieving earlier availability of angiography or an effective alternative treatment for pelvic fracture related hemorrhage should decrease mortality.

45.• Morozumi J, et al. Introduction of mobile angiography into the trauma resuscitation room. J Trauma. 2009;67(2):245-51. A before and after study that shows how training trauma surgeons to perform angioembolization allows this modality to be used immediately to treat hemorrhagic shock, thereby achieving hemostasis earlier and decreasing mortality.

46. Morozumi J, et al. Impact of mobile angiography in the emergency department for controlling pelvic fracture hemorrhage with hemodynamic instability. J Trauma. 2010;68(1):90-5.

47. Verbeek D, et al. Acute management of hemodynamically unstable pelvic trauma patients: time for a change? Multicenter review of recent practice. World J Surg. 2008;32(8):1874-82.

48. Bryceland JK, Keating JF. Laparotomy and unstable pelvic fractures. Injury. 2008;39(8):853-7.

49. Ruchholtz S, et al. Free abdominal fluid on ultrasound in unstable pelvic ring fracture: is laparotomy always necessary? J Trauma. 2004;57(2):278-85. discussion 285-7.

$50 . \bullet$ Verbeek DO, et al. Predicting the need for abdominal hemorrhage control in major pelvic fracture patients: the importance of quantifying the amount of free fluid. J Trauma Acute Care Surg. 2014;76(5):1259-63. This paper describes a simple method to quantify the amount of free intraperitoneal fluid on a CT scan and demonstrates how it can accurately predict the need for laparotomy in patients with major pelvic fractures and hemorrhagic shock.

51. Charbit J, et al. Does the size of the hemoperitoneum help to discriminate the bleeding source and guide therapeutic decisions in blunt trauma patients with pelvic ring fracture? J Trauma Acute Care Surg. 2012;73(1):117-25.

52. Hagiwara A, et al. Predictors of death in patients with lifethreatening pelvic hemorrhage after successful transcatheter arterial embolization. J Trauma. 2003;55(4):696-703.

53. Bize PE, et al. Embolization for multicompartmental bleeding in patients in hemodynamically unstable condition: prognostic factors and outcome. J Vasc Interv Radiol. 2012;23(6):751-760 e4. This paper demonstrates the degree of succes that can be expected when attempting to control multiple sources of hemorrhage with angioembolization.

54. Fangio P, et al. Early embolization and vasopressor administration for management of life-threatening hemorrhage from pelvic fracture. J Trauma : Inj Infect Crit Care. 2005;58(5):978-84.

55. Eastridge BJ, et al. The importance of fracture pattern in guiding therapeutic decision-making in patients with hemorrhagic shock and pelvic ring disruptions. J Trauma. 2002;53(3):446-50. discussion $450-1$.
56. Fang JF, et al. Angioembolization and laparotomy for patients with concomitant pelvic arterial hemorrhage and blunt abdominal trauma. Langenbecks Arch Surg. 2011;396(2): 243-50.

57. Cook RE, Keating JF, Gillespie I. The role of angiography in the management of haemorrhage from major fractures of the pelvis. J Bone Joint Surg Br. 2002;84(2):178-82.

58. DuBose $\mathrm{J}$, et al. Bilateral internal iliac artery ligation as a damage control approach in massive retroperitoneal bleeding after pelvic fracture. J Trauma. 2010;69(6):1507-14.

59. Cothren CC, et al. Preperitonal pelvic packing for hemodynamically unstable pelvic fractures: a paradigm shift. J Trauma. 2007;62(4): 834-9. discussion 839-42.

60. Thorson CM, et al. Operating room or angiography suite for hemodynamically unstable pelvic fractures? J Trauma Acute Care Surg. 2012;72(2):364-70. discussion 371-2.

61. Cherry RA, et al. Intraoperative angioembolization in the management of pelvic-fracture related hemodynamic instability. J Trauma Manag Outcomes. 2011;5:6.

$62 . \bullet$ Kirkpatrick AW, et al. The evolution of a purpose designed hybrid trauma operating room from the trauma service perspective: the RAPTOR (resuscitation with angiography percutaneous treatments and operative resuscitations). Injury. 2014;45(9):1413-21. This paper describes optimal design of a hybrid trauma operating room. This is the optimal environment to conduct multidisciplinary care of patients with pelvic fracture related hemorrhage.

63. Teo LT, et al. Emergency angio-embolisation in the operating theatre for trauma patients using the C-Arm digital subtraction angiography. Injury. 2012;43(9):1492-6.

64. Margolies MN, et al. Arteriography in the management of hemorrhage from pelvic fractures. N Engl J Med. 1972;287(7):317-21.

65. Ring EJ, et al. Arteriographic management of hemorrhage following pelvic fracture. Radiology. 1973;109(1):65-70.

66. Ayella RJ, et al. Transcatheter embolization of autologous clot in the management of bleeding associated with fractures of the pelvis. Surg Gynecol Obstet. 1978;147(6):849-52.

67. Matalon TS, et al. Hemorrhage with pelvic fractures: efficacy of transcatheter embolization. AJR Am J Roentgenol. 1979;133(5): 859-64.

68. Lang EK. Transcatheter embolization of pelvic vessels for control of intractable hemorrhage. Radiology. 1981;140(2):331-9.

69. Yellin AE, Lundell CJ, Finck EJ. Diagnosis and control of posttraumatic pelvic hemorrhage. Transcatheter angiographic embolization techniques. Arch Surg. 1983;118(12):1378-83.

70. Velmahos GC, et al. Angiographic embolization of bilateral internal iliac arteries to control life-threatening hemorrhage after blunt trauma to the pelvis. Am Surg. 2000;66(9):858-62.

71. van der Vlies $\mathrm{CH}$, et al. Failure rate and complications of angiography and embolization for abdominal and pelvic trauma. J Trauma Acute Care Surg. 2012;73(5):1208-12.

72. Ramirez JI, et al. Male sexual function after bilateral internal iliac artery embolization for pelvic fracture. J Trauma : Inj Infect Crit Care. 2004;56(4):734-41.

73. Travis T, et al. Evaluation of short-term and long-term complications after emergent internal iliac artery embolization in patients with pelvic trauma. J Vasc Interv Radiol. 2008;19(6): 840-7.

74. Auerbach AD, Rehman S, Kleiner MT. Selective transcatheter arterial embolization of the internal iliac artery does not cause gluteal necrosis in pelvic trauma patients. J Orthop Trauma. 2012;26(5): 290-5.

75. Yasumura $\mathrm{K}$, et al. High incidence of ischemic necrosis of the gluteal muscle after transcatheter angiographic embolization for severe pelvic fracture. J Trauma : Inj Infect Crit Care. 2005;58(5):985-90. 
76. Matityahu A, et al. Acute complications of patients with pelvic fractures after pelvic angiographic embolization. Clin Orthop Relat Res. 2013;471(9):2906-11.

77. Saour M, et al. Effect of renal angioembolization on post-traumatic acute kidney injury after high-grade renal trauma: a comparative study of 52 consecutive cases. Injury. 2014;45(5):894-901.

78. Vassiliu P, et al. Is contrast as bad as we think? Renal function after angiographic embolization of injured patients. J Am Coll Surg. 2002;194(2):142-6.

79. Yuan KC, et al. Delayed hemorrhage caused by coil migration after transcatheter arterial embolization in patient with unstable pelvic fracture: a case report. J Trauma. 2009;66(1): $267-70$.
80. Gourlay D, et al. Pelvic angiography for recurrent traumatic pelvic arterial hemorrhage. J Trauma : Inj Infect Crit Care. 2005: 1168-1174.

81. Shapiro M, et al. The role of repeat angiography in the management of pelvic fractures. J Trauma : Inj Infect Crit Care. 2005;58(2):227-31.

82. Fang JF, et al. Repeat transcatheter arterial embolization for the management of pelvic arterial hemorrhage. J Trauma. 2009;66(2): 429-35.

83. Geeraerts T, Chhor V, Cheisson G, Martin L, Bessoud B, Ozanne A, Duranteau J. Clinical review: initial management of blunt pelvic trauma patients with haemodynamic instability. Crit Care. 2007; 11(1):204 